This article may be downloaded for personal use only. Any other use requires prior permission of the author and AIP Publishing. This article appeared in Appl. Phys. Lett. 113, 092901 (2018) and may be found at https://aip.scitation.org/doi/10.1063/1.5046080

\title{
Electromechanical control of polarization vortex ordering in an interacting ferroelectric-dielectric composite dimer
}

\author{
John Mangeri, ${ }^{1,2, a)}$ S. Pamir Alpay, ${ }^{2,3}$ Serge Nakhmanson, ${ }^{2,3}$ and Olle G. Heinonen ${ }^{4,5}$ \\ ${ }^{1)}$ Institute of Physics, Czech Academy of Sciences, Na Slovance 2, 18221 Prague 8, Prague, Czech Republic \\ ${ }^{2)}$ Department of Physics, University of Connecticut, Storrs, Connecticut 06269, USA \\ ${ }^{3)}$ Department of Materials Science \& Engineering, and Institute of Materials Science, University of Connecticut, Storrs, \\ Connecticut 06269, USA \\ 4) Material Science Division, Argonne National Laboratory, Lemont, Illinois 60439, USA \\ ${ }^{5)}$ Center for Hierarchical Material Design, Northwestern-Argonne Institute of Science and Engineering, \\ Northwestern University, Evanston, Illinois 60201, USA
}

(Dated: June 25, 2018)

\begin{abstract}
Using a free-energy based computational model, we have investigated the response of a system comprised of two interacting ferroelectric nanospheres, embedded in a dielectric medium, to a static external electric field. The system response is hysteretic and tunable by changing the inter-particle distance and the orientation of the applied field, which strongly modulates the field-driven long-range elastic interactions between the particles that propagate through the dielectric matrix. At small separations, the sensitivity of the system behavior with respect to the electric field direction originates from drastically different configurations of the local vortex-like polarization states in the ferroelectric particles. This suggests new routes for the design of composite metamaterials whose dielectric properties can be controlled and tuned by selecting the mutual arrangement of their ferroelectric components.
\end{abstract}

Ferroelectric-dielectric composite systems display a wide range of functional behavior. For particulate dispersed phases, precise control of the inter-particle spacing and arrangement ${ }^{1}$, particle shape morphology 23, surface crystallography and concentration $\frac{5}{5}$, as well as selection of materials for both phases (with the matrix being of ceramic 67 or polymeric origin ${ }^{15 / 8 \mid 9}$ ), can lead to designs with preprogrammed properties and novel functionalities. In particular, remarkable behavioral features, such as superparaelectricity $\sqrt{8}$, enhanced dielectric tunability ${ }^{10}$ and energy storage density ${ }^{8 / 11 / 12}$, multi-state ferroelectric (FE) switching ${ }^{13 \mid 14}$, and reduced conductivity and loss $\frac{67}{6}$ were already demonstrated in ferroelectric-dielectric composites, which makes these systems attractive for numerous future technological applications 15 .

A number of synthetic techniques have been recently used to fabricate nanoparticles of binary $\sqrt{16 / 18}$ and ternary $\sqrt{3 / 19}[22$ oxides in a variety of shapes and sizes. The properties of these particles, as well as the nature of FE ordering in them, have been characterized with multiple experimental approaches, including Bragg coherent diffraction imaging $\sqrt{23}$ and second harmonic generation microscopy $\sqrt{24 \mid 26}$ for sizes of above 50-60 $\mathrm{nm}$, and aberration corrected transmission electron microscopy (TEM) ${ }^{2728}$ and local piezoforce microscopy for particles of to $5-15 \mathrm{~nm}$ in size $27 / 29$. Both monodomain ${ }^{27}$ and vortexlike $e^{14|23| 29}$ polarization topologies were observed in the FE particles, with the latter patterns expected to be utilized in memory elements for new paradigms in computing 1330 .

Formation of topological polarization patterns in isolated FE particles has also been studied with different coarse-grained theoretical approaches, such as phase-field ${ }^{12|28| 31 \mid 32}$, semianalytic 33 , 36 and effective hamiltonian $\sqrt[37 / 38]{ }$ methods. For example, in previous work ${ }^{32139}$, we evaluated size and shape dependent phase transitions in individual FE nanoparticles embedded in a dielectric matrix, observing a monodomain to vortex-like to polydomain sequence of transformations for the increasing particle size. However, in a composite system, this situation corresponds to a highly dispersed arrangement of particles within the matrix, with negligible electrostatic and elastic interactions between them. On the other hand, it is expected that more promising functional behavior can be obtained in complex systems consisting of many interacting particles, the aggregate responses of which should be heavily influenced by the particle size, shape, anisotropy and mutual arrangement ${ }^{1 / 40}$.

As accurate treatment of composite materials including numerous interacting FE particles presents a formidable computational challenge even on the coarse-grained level of theory, the next step towards developing a better understanding of such compounds involves investigation of systems with few interacting particles to contrast their behavior against the single-particle case. Here, using the time-dependent LandauGinzburg-Devonshire (TDLGD) approach ${ }^{3241}$, we explore a dimer system, consisting of two $\mathrm{PbTiO}_{3}$ (PTO) nanospheres embedded in a linear dielectric medium represented by $\mathrm{SrTiO}_{3}$ (STO). Our main goals include determination of a zero-field polarization pattern within the dimer as a function of the interparticle separation $\eta$, followed by evaluation of the quasi-static response of the dimer system to an electric field that can be applied in different directions (as shown in the top panel of Fig. 1). Throughout this study, both particle diameters are kept fixed at $d=10 \mathrm{~nm}$ : the size identified in our previous investigation ${ }^{32}$ as the one that supports a zero electric field vortex-like polarization state.

Following the TDLGD approach, the Cartesian components $k=1,2,3$ of the local polarization field, $\mathbf{P}(\mathbf{r})$, are evolved in 
time as

$$
\frac{\partial P_{k}}{\partial t}=-\Gamma \frac{\delta \mathcal{F}}{\delta P_{k}},
$$

where $\mathcal{F}$ is the total electric enthalpy of the composite, and $\Gamma$ is a coefficient related to the FE domain wall mobility 4 . Detailed expressions for all of the energy terms included in $\mathcal{F}$ are provided in the Supplemental Materials (SM), together with the utilized materials parameters for PTO and STO.

We assume that during the system time evolution, elastic strains relax at much faster time-scales than $\mathbf{P}^{4143}$. Therefore, at each time step, for fixed polarization, the strain fields $\varepsilon_{i j}=$ $\frac{1}{2}\left(\partial u_{i} / \partial x_{j}+\partial u_{j} / \partial x_{i}\right)$ can be obtained from the mechanical equilibrium equation

$$
\frac{\partial}{\partial x_{j}} C_{i j k l}\left(\varepsilon_{k l}-Q_{k l m n} P_{m} P_{n}\right)=0,
$$

where $C_{i j k l}$ and $Q_{i j k l}$ are the elastic stiffness and electrostrictive tensor components, respectively, and $\mathbf{u}(\mathbf{r})$ is the elastic displacement field. The electrostatic potential $\Phi(\mathbf{r})$ is computed from the Poisson equation

$$
\frac{\partial}{\partial x_{j}}\left(\epsilon_{b} \frac{\partial \Phi}{\partial x_{j}}\right)=\frac{\partial P_{k}}{\partial x_{k}},
$$

where $\epsilon_{b}$ is the background dielectric tensor ${ }^{44}$, assumed to be isotropic.

We solve Eqs. (1-3) using finite-element methods within the open-source MOOSE framework ${ }^{[5]}$ and the FERRET module ${ }^{46}$ developed by the authors.

In the absence of an applied external electric field, the boundary conditions on the six sides of the system volume (chosen to be sufficiently large with the dimer at its center) are $\mathbf{u}=0$, and $\Phi=0$. In the presence of an applied $\mathbf{E}$ field, a potential difference $\Delta \Phi=\Phi_{1}-\Phi_{2}$ is applied to two opposing sides of the box. The initial condition on $\mathbf{P}(\mathbf{r})$ is chosen as a random field of small local polarizations such that $\langle\mathbf{P}\rangle \approx 0$, i.e., mimicking the high-temperature paralectric state. As $\mathbf{P}$ is evolved in time [Eq. (1)], energy is dissipated from the system, with the simulation terminated when the relative change in $\mathcal{F}$ between consecutive time steps is approximately $0.01 \%$.

An example of a dimer equilibrium state for $\eta=10 \mathrm{~nm}$ under the $\mathbf{u}, \Phi \rightarrow 0$ boundary conditions is presented in Fig. 1 The polarization field aligns tangentially to the particle surface to minimize surface charges, creating a vortex-like pattern in each of the two spheres. Such an equilibrium structure is different from the flux-closure points typically observed at intersections of four $90^{\circ} \mathrm{FE}$ domain walls $31 / 47$. The pattern of Fig. 1 has diffuse domain walls, with the $\mathbf{P}(\mathbf{r})$ distribution continuously rotating about a central axis ${ }^{23 \mid 32}$ represented by an axial director $\mathbf{v}$. The cylindrical vortex-core region penetrates through the sphere and usually has polarization magnitudes that are lower than at the surfaces. This behavior is different from that of ferromagnetic vortices, in which the magnetization density magnitude is constant ${ }^{4849}$ well below $T_{\mathrm{C}}$.

For inter-particle spacings $\eta \lesssim 10 \mathrm{~nm}$, the core-region orientations in the two particles can become strongly correlated. Specifically, the angles between the directors $\mathbf{v}$ of the two spheres can adopt a discrete set of values, suggesting that certain mutual core-region orientations within the dimer are more energetically preferred than others. In contrast with the single particle case, in some instances we also observe the development of large polarization, comparable to the surface values, in the vortex-core regions of both spheres. Some examples of such polarization patterns are shown in the SM for $\eta=2 \mathrm{~nm}$. For large inter-particle distances $\eta \gtrsim 10 \mathrm{~nm}$, we observe no pronounced correlations between the core-region orientations of the dimer particles; the orientation of the director $\mathbf{v}$ in each sphere depends mostly on the specific configuration of $\mathbf{P}(\mathbf{r})$ generated by the random initial condition. Therefore, in the absence of an externally applied $\mathbf{E}$, the dimer particles do not strongly interact with each other: the stray fringing electric fields from the surface and volume charge distributions of each sphere are effectively screened by the high dielectric permittivity $\left(\epsilon_{b}=300 \epsilon_{0}\right)$ of the surrounding matrix, and the strain fields propagating from the surface of each sphere into the matrix decay within a short distance.

We next turn to the analysis of the quasi-static dimer $P-E$ curves $^{32 \mid 50}$. In the previous study, the $P-E$ loops of an isolated PTO sphere with $d=10 \mathrm{~nm}$ embedded in STO were found to exhibit shelves of roughly constant $\overline{P_{E}}=\overline{\mathbf{P} \cdot \hat{\mathbf{E}}}$ at smaller applied fields ( $\bar{X}$ denotes volume-average of a scalar field $X$ ), which was associated with the presence of a vortex-like phase that transformed into a uniform monodomain phase when the field was increased, as shown in the insert of Fig.2.b). A useful measure of the system vorticity is the FE-volume-averaged winding number density $\xi=|\mathbf{P} \cdot(\nabla \times \mathbf{P})| ; \xi \approx 0$ in the monodomain phase, and saturates to a constant value in the

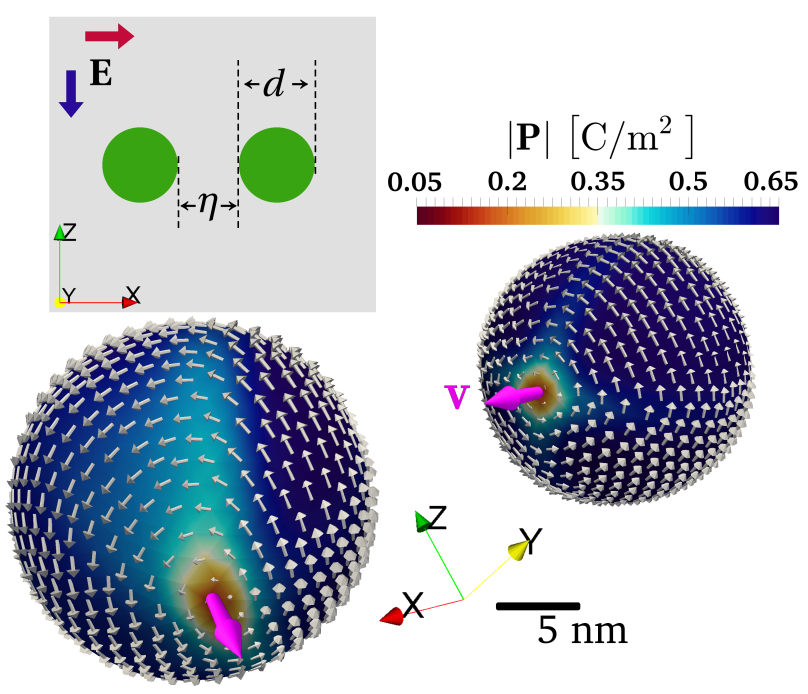

FIG. 1. Top panel: diagram (not to scale) of the interacting PTO particle dimer of diameter $d$ separated by a surface-to-surface distance $\eta$, embedded in a matrix of a linear elastic dielectric (here STO). The electric field directions are shown as blue $(\hat{\mathbf{E}}|| \hat{\mathbf{z}})$ or red $(\hat{\mathbf{E}}|| \hat{\mathbf{x}})$ arrows, with the latter being parallel to the axis connecting the particles. The bottom panel shows a typical weakly-correlated polarization vortexlike state of the dimer with $\eta=10 \mathrm{~nm}$ after an energy minimization in zero applied field. Director $\mathbf{v}$ (pink arrow) is an axial vector representing the orientation of the cylindrical polar-vortex core. 


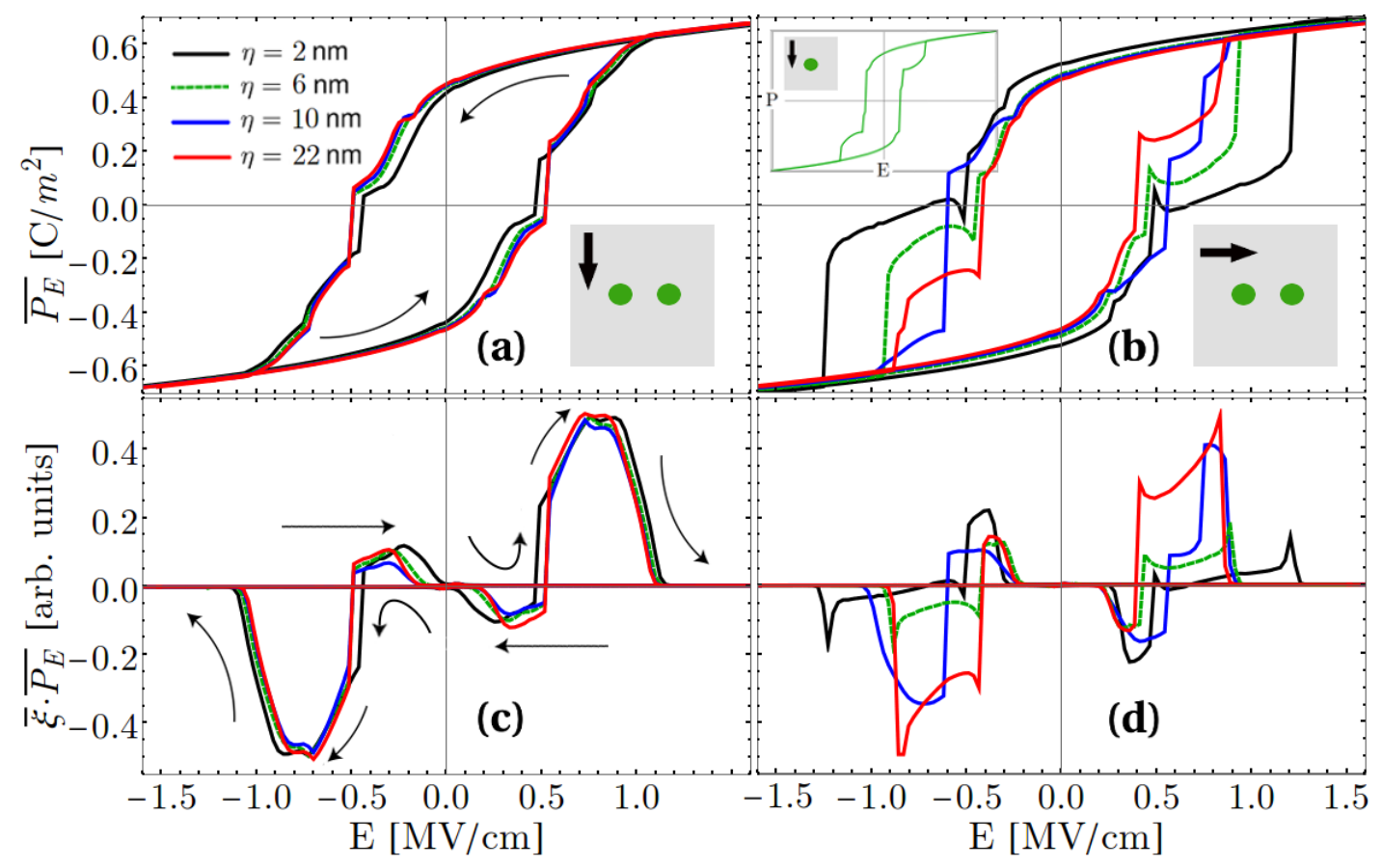

FIG. 2. $P$ - $E$ loops for the averaged polarization component along the direction of the applied electric field $P_{E}$ for (a): $\hat{\mathbf{E}}|| \hat{\mathbf{z}}$ and (b): $\hat{\mathbf{E}}|| \hat{\mathbf{x}}$, and $\eta=2 \mathrm{~nm}$ (black), $6 \mathrm{~nm}$ (green), $10 \mathrm{~nm}$ (blue), and $22 \mathrm{~nm}$ (red). Panels (c) and (d) depict the magnitude of the averaged winding number density $\xi$ weighted with the average polarization along the field $P_{E}$ as a function of the electric field for the configurations (a) and (b), respectively. The upper inset in (b) shows the $P-E$ loop of a single sphere with $d=10 \mathrm{~nm}$ calculated with the same approach (from Ref. 32).

polydomain phase, however, in the adjoining vortex-like phase region it goes through a sharp maximum 32 .

The $P-E$ loops presented in Fig. 2(a) and (b) are computed for $\eta=2,6,10$, and $22 \mathrm{~nm}$ for both orientations of the applied electric field shown in the top panel of Fig. 1. For each point of the loop, the components of local $P_{E}(\mathbf{r})$ are averaged over both spheres. The averaged winding number density $\bar{\xi}$ is presented in panels (c) and (d) of Fig. 2 weighted with the average polarization along the field $\overline{P_{E}}$ as a function of the applied field magnitude $E$.

The orientation of the applied field relative to the dimer axis profoundly influences the shape of the $P-E$ response. With the field perpendicular to the axis connecting the particles $(\hat{\mathbf{E}} \| \hat{\mathbf{z}})$ [Fig. reffig:hyst(a) and (c)], both the $\overline{P_{E}}$ and $\bar{\xi} \cdot \overline{P_{E}}$ dependencies exhibit only minor changes with increasing inter-particle spacing $\eta$. In particular, the transition between monodomain and vortex-like states occurs at the same value of $E \approx 0.2$ $0.3 \mathrm{MV} / \mathrm{cm}$ for all values of $\eta$. As the applied field switches sign, a small domain of correlated $\mathbf{P}$ along the direction of $\mathbf{E}$ begins to nucleate within the vortex-like phase in each sphere. With the increasing strength of the applied field, these domains grow until reaching saturation, which results in a gradual decrease and then complete disappearance of $\bar{\xi}$. This behavior seems to be universal and not strongly dependent on the value of inter-particle spacing $\eta$.

When the field is applied along the axis connecting the particles $(\hat{\mathbf{E}}|| \hat{\mathbf{x}})$, as shown in panels (b) and (d) of Fig. 2 a radically different system behavior is observed, as manifested by the appearance of shelf-like features in both $\overline{P_{E}}(E)$ and
$\bar{\xi}(E)$ curves, indicating the presence of the vortex-like phase. The transition into the vortex-like state happens at the same value of $E$ as in the previous case, i.e., $0.2-0.3 \mathrm{MV} / \mathrm{cm}$. The width of the shelf-like feature on the $\overline{P_{E}}(E)$ curve is roughly inversely proportional to the value of $\eta$, as can be seen from the comparison of the loops for $\eta=2 \mathrm{~nm}$ and $\eta=6 \mathrm{~nm}$ [see Fig. 2 (b)]. The $\bar{\xi}(E)$ dependence exhibits sharp peaks on both ends of the shelf that are especially pronounced for $\eta=2 \mathrm{~nm}$ and $\eta=6 \mathrm{~nm}$ curves and can serve as good indicators for an onset of a phase transition. Inspection of the corresponding $\mathbf{P}(\mathbf{r})$ distributions shows that these peaks arise during a formation of multiple vortex-like patterns in each sphere. These patterns then merge into a single vortex with a core direction aligned with the direction of the field; some images illustrating the process are presented in the SM. For $\eta \gtrsim 20 \mathrm{~nm}$, the system $P-E$ loops become similar to that of an isolated sphere of identical size, cf. the $\eta=22 \mathrm{~nm}$ curve and the curve in the inset of Fig. 2(b).

In order to better understand the origins of the varying dimer system response under different orientations of the applied field we examine the behavior of elastic strain fields as a function of the direction of E. In Fig. 3, color maps of the diagonal components of the elastic strain tensor $\varepsilon_{i i}$ along the field are presented for the case of inter-particle separation of $6 \mathrm{~nm}$ and the field magnitude of $1.0 \mathrm{MV} / \mathrm{cm}$ applied in the $-\hat{\mathbf{z}}$ direction (left panel) and $-\hat{\mathbf{x}}$ direction (right panel). At this electric field strength, both particles in each configuration are in the monodomain state, with the polarization parallel to the applied field. Due to the nearly constant polarization, the elastic strain inside each sphere along the field direction $i$ is tensile, 


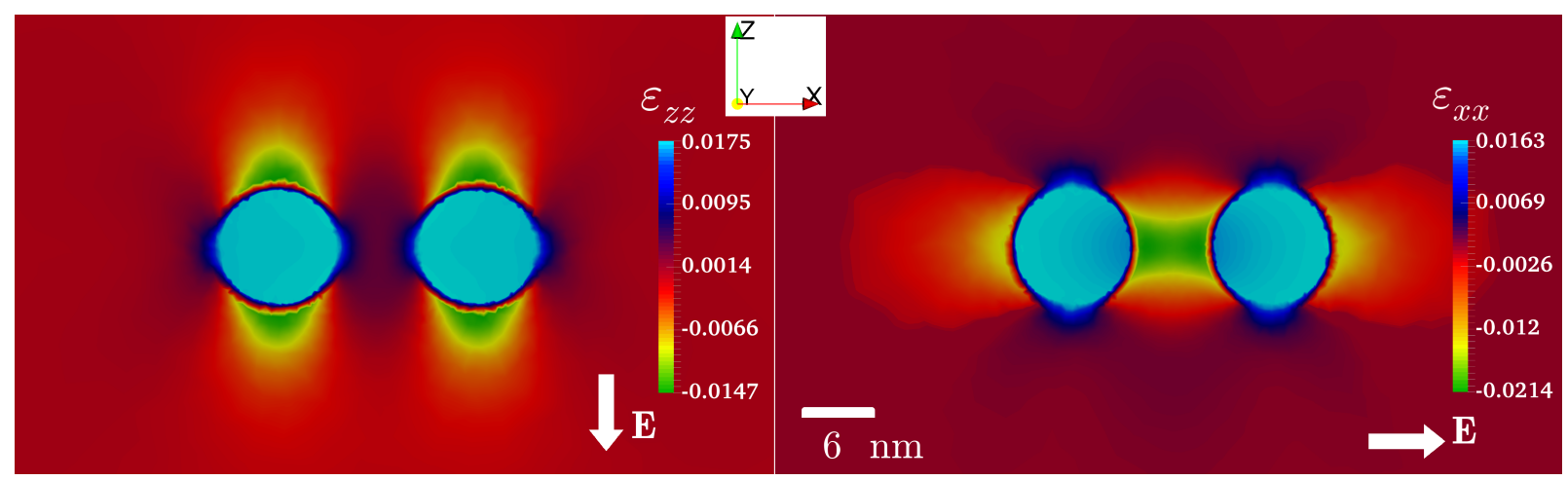

FIG. 3. Color maps of the diagonal components of the $\varepsilon_{i i}$ in the $x-z$ plane in the dimer separated by $\eta=6 \mathrm{~nm}$, with the field $E=$ $1.0 \mathrm{MV} / \mathrm{cm}$ applied along $-\hat{\mathbf{z}}$ (left panel) and $-\hat{\mathbf{x}}$ (right panel). The tensile strain is nearly constant inside the spheres, which is consistent with a constant-polarization state along the applied electric field direction. As the spheres expand slightly into the matrix, it develops compressed regions that are disconnected when $\hat{\mathbf{E}}|| \hat{\mathbf{z}}$ (left panel) and joined when $\hat{\mathbf{E}}|| \hat{\mathbf{x}}$ (right panel). Note the different color bar scales in the panels.

$\varepsilon_{i i} \approx 0.0165 \%$. Therefore, the matrix region in between the particles experiences tensile strain for $\hat{\mathbf{E}} \mid \mathbf{z}$ and compressive strain for $\hat{\mathbf{E}} \mid \hat{\mathbf{x}}$, as shown, respectively, in the left and right panels of Fig. 3. Elastic distortions extend for $\sim 10 \mathrm{~nm}$ from the sphere surfaces into the matrix and decay to zero at the boundaries of the computational domain (see SM for an example). The strain transfer mechanism in the composite arises from the electromechanical coupling between the FE particles and the dielectric medium: the applied electric field elongates the particles slightly along the direction of the field, which in turn causes elastic distortions within the matrix.

For the $\hat{\mathbf{E}}|| \hat{\mathbf{x}}$ case, the strain in the region between the spheres is approximately additive for small separations $\eta$, which leads to a strong elastic coupling between the spheres. In turn, this results in a substantial decrease of $\overline{P_{E}}$, compared to the $\hat{\mathbf{E}} \| \hat{\mathbf{z}}$ case: e.g., $\overline{P_{E}} \equiv \overline{P_{z}}=0.35$ vs. $\overline{P_{E}} \equiv \overline{P_{x}}=0.20$ $\mathrm{C} / \mathrm{m}^{2}$ for $\eta=6 \mathrm{~nm}$ and $E=0.7 \mathrm{MV} / \mathrm{cm}$, as shown in panels (a) and (b), respectively, of Fig. 2 The origin of this difference stems from contrasting orientations of the particle vortex cores with respect to $\mathbf{E}$, as shown in Fig. 4. In the $\hat{\mathbf{E}} \| \hat{\mathbf{z}}$ case, shown in panel (a), core orientation vectors $\mathbf{v}$ of both spheres are perpendicular to $\mathbf{E}$ and it is the large local polarization from the surface regions that contributes to the average value of $P_{E}$. In contrast, in the $\hat{\mathbf{E}}|| \hat{\mathbf{x}}$ case, shown in panel (b), the directors $\mathbf{v}$ of both spheres are aligned along $\mathbf{E}$, with only the core-region polarization, which is greatly suppressed compared to the surface regions contributing to $\overline{P_{E}}$. Therefore, it is the $\mathbf{v}\|\hat{\mathbf{E}}\| \hat{\mathbf{x}}$ system orientation that gives rise to the shelf-like features with depressed near-constant $\overline{P_{E}}$ in Fig. 2(b) at small inter-particle separations. As the electric field increases, the core-region polarization saturates and then proliferates in the $y-z$ plane until a monodomain state is formed and vorticity vanishes.

We have investigated the influence dimer size and electric field orientation on the ordering and response of a FE-dielectric composite system. Our work demonstrates that the dimer size and electric field direction can be used to control the dielectric response in such composite systems, with potential applications in electronic and elecctromechanical devices.

\section{SUPPLEMENTAL MATERIAL}

See supplemental material for additional details on the calculations and supporting information.

\section{ACKNOWLEDGMENTS}

JM acknowledges funding under the Ministry of Education, Youth and Sports of the Czech Republic, grant number CZ.02.2.69/0.0/0.0/16_027/0008215. OH was funded by the Department of Energy, Office of Science Basic Energy Sciences Division of Materials Science and Engineering. JM is grateful to A. Jokisaari for assistance with calculations.

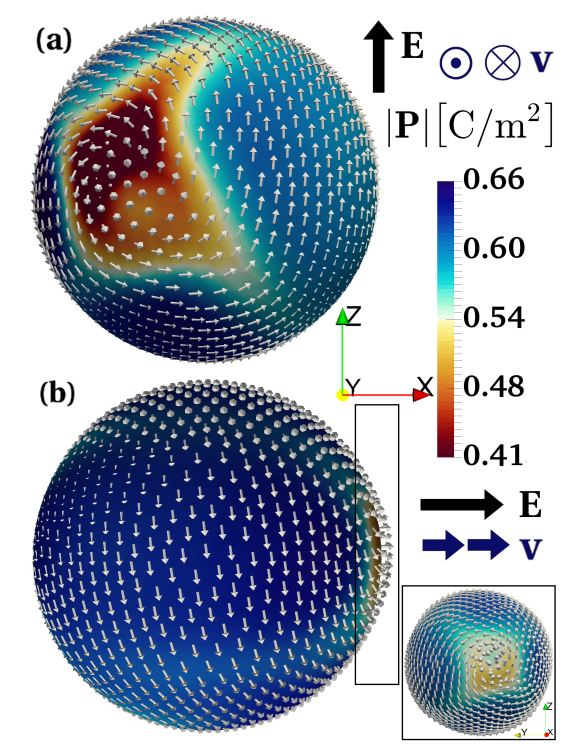

FIG. 4. $\quad \mathbf{P}(\mathbf{r})$ patterns in one of the dimer particles for $\eta=6$ $\mathrm{nm},|E|=0.7 \mathrm{MV} / \mathrm{cm}$ and (a) $\hat{\mathbf{E}}|| \hat{\mathbf{z}}$, or (b) $\hat{\mathbf{E}}|| \hat{\mathbf{x}}$. The depressed magnitude of the core-region polarization is highlighted by the color map. The inset to panel (b) shows the vortex-like $\mathbf{P}(\mathbf{r})$ pattern with $\mathbf{v} \| \mathbf{E}$. 


\section{REFERENCES}

${ }^{a}$ Electronic mail: mangeri@fzu.cz

${ }^{1}$ J. Feng, Q. Song, B. Zhang, Y. Wu, T. Wang, and L. Jiang, Adv. Mater. 29, 1703143 (2017).

${ }^{2}$ Y. Hao, X. Wang, and L. Li, Nanoscale 6, 7940 (2014).

${ }^{3}$ D. Caruntu, T. Rostamzadeh, T. Costanzo, S. S. Parizi, and G. Caruntu, Nanoscale 7, 12955 (2015).

${ }^{4}$ X. Wang, Y. Yan, T. Shimada, J. Wang, and T. Kitamura, J. Appl. Phys. 123 (2018).

${ }^{5}$ Y. N. Hao, K. Bi, S. O'Brien, X. X. Wang, J. Lombardi, F. Pearsall, W. L. Li, M. Lei, Y. Wu, and L. T. Li, RSC Adv. 7 (2017).

${ }^{6}$ M. Airimioaei, M. T. Buscaglia, I. Tredici, U. UnselmiTamburini, C.-E. Ciomaga, L. Curecheriu, V. Buscaglia, and L. Mitoseriu, J. Mater. Chem. C. 5, 9028 (2017).

${ }^{7}$ E. A. Nenasheva, N. F. Kartenko, I. M. Gaidamaka, S. S. Redozubov, A. B. Kozyrev, and A. D. Kanareykin, Ferroelectrics 506:1, 174 (2017).

${ }^{8}$ L. Huang, Z. Jia, I. Kymissis, and S. O'Brien, Adv. Func. Mater. 20, 554 (2010).

${ }^{9}$ S. A. Paniagua, Y. Kim, K. Henry, R. Kumar, J. W. Perry, and S. R. Marder, ACS. Appl. Mater. Interfaces 6, 3477 (2014).

${ }^{10}$ K. Zhou, S. A. Boggs, R. Ramprasad, M. Aindow, C. Erkey, and S. P. Alpay, Appl. Phys. Lett. 93, 102908 (2008).

${ }^{11}$ X. Zhang, Y. Shen, Q. Zhang, L. Gu, Y. Hu, J. Du, Y. Lin, and C.-W. Nan, Adv. Mater. 27, 819 (2014).

${ }^{12}$ Z. Liu, B. Yang, W. Cao, E. Fohtung, and T. Lookman, Phys. Rev. Appl. 8, 034014 (2017).

${ }^{13}$ D. Gruverman, A. Wu, H.-J. Fan, I. Vrejoiu, M. Alexe, R. J. Harrison, and J. F. Scott, J. Phys.: Condens. Matter 20, 342201 (2008).

${ }^{14}$ Z. Li, Y. Wang, G. Tian, P. Li, L. Zhao, F. Zhang, J. Yao, H. Fan, X. Song, D. Chen, Z. Fan, M. Qin, M. Zeng, Z. Zhang, X. Lu, S. Hu, C. Lei, Q. Zhu, J. Li, X. Gao, and J.-M. Liu, Sci. Adv. 3, e1700919 (2017).

${ }^{15} \mathrm{P}$. Alitalo and S. Tretyakova, Materials Today 12, 22 (2009).

${ }^{16}$ N. R. Jana, Y. Chen, and X. Peng, Chem. Mater. 16, 3931 (2004).

${ }^{17}$ Y.-W. Jun, J.-S. Choi, and J. Cheon, Angew. Chem. Int. Ed. 45, 3414 (2006).

${ }^{18}$ W.-X. Tang and P.-X. Gao, MRS Commun. 6, 311 (2016).

${ }^{19}$ T.-J. Park, G. C. Papaefthymiou, A. R. Moodenbaugh, Y. Mao, and S. S. Wong, J. Mater. Chem. 15, 2099 (2005).

${ }^{20}$ T. A. Tyson, T. Yu, M. Croft, M. E. Scofield, D. BobbSemple, J. Tao, C. Jaye, D. Fischer, and S. S. Wong, Appl. Phys. Lett. 105 (2014).

${ }^{21}$ G. C. Papaefthymiou, A. J. Viescas, J.-M. L. Breton, H. Chiron, J. Juraszek, T.-J. Park, and S. S. Wong, Curr. Appl. Phys. 15, 417 (2015)

${ }^{22}$ H. Zhang, S. Liu, M. E. Scofield, S. S. Wong, X. Hong, V. B. Prakapenka, E. Greenberg, and T. A. Tyson, Appl. Phys. Lett. 111, 052904 (2017)

${ }^{23}$ D. Karpov, Z. Liu, T. dos Santos Rolo, R. Harder, P. V. Balachandran, D. Xue, T. Lookman, and E. Fohtung, Nat. Comm. 8, 280 (2017).
${ }^{24}$ S. Cherifi-Hertel, H. Bulou, R. Hertel, G. Taupier, K. D. H. Dorkenoo, C. Andreas, J. Guyonnet, I. Gaponenko, K. Gallo, and P. Paruch, Nature Commun. 8, 15768 (2017).

${ }^{25}$ C. Ma, J. Yan, Y. Wei, P. Liu, and G. Yang, J. Mater. Chem. C 5, 4810 (2017).

${ }^{26}$ F. Timpu, A. Sergeyev, N. R. Hendricks, and R. Grange, ACS Photonics 4, 76 (2017).

${ }^{27}$ M. J. Polking, M.-G. Han, A. Yourdkhani, V. Petkov, C. F. Kisielowski, V. V. Volkov, Y. Zhu, G. Caruntu, A. P. Alivisatos, and R. Ramesh, Nature Materials 11, 700 (2012).

${ }^{28}$ A. K. Yadav, C. T. Nelson, S. L. Hsu, Z. Hong, J. D. Clarkson, C. M. Schlepütz, A. R. Damodaran, P. Shafer, E. Arenholz, L. R. Dedon, D. Chen, A. Vishwanath, A. M. Minor, L.-Q. Chen, J. F. Scott, L. W. Martin, and R. Ramesh, Nature 530, 198 (2016).

${ }^{29}$ B. J. Rodriguez, X. S. Gao, L. F. Liu, W. Lee, I. I. Naumov, A. M. Bratkovsky, D. Hesse, and M. Alexe, Nano Lett. 9, 1127 (2009).

${ }^{30}$ J. F. Scott, Science 315, 954 (2007).

${ }^{31}$ C. M. Wu, W. J. Chen, Y. Zheng, D. C. Ma, B. Wang, J. Y. Liu, and C. H. Woo, Sci. Rep. 4 (2014).

${ }^{32}$ J. Mangeri, Y. Espinal, A. Jokisaari, S. P. Alpay, S. Nakhmanson, and O. Heinonen, Nanoscale 9, 1616 (2017).

${ }^{33}$ E. K. Akdogan and A. Safari, J. Appl. Phys. 101, 064114 (2007).

${ }^{34}$ M. D. Glinchuk, E. A. Eliseev, and A. N. Morozovska, Phys. Rev. B. 78, 134107 (2008).

${ }^{35}$ P.-W. Martelli, S. Mefire, and I. A. Luk'Yanchuk, Euro. Phys. Lett. 111, 50001 (2015).

${ }^{36}$ R. Qiu, E. Bousquet, and A. Cano, Euro. Phys. Lett. 112, 37006 (2015).

${ }^{37}$ I. I. Naumov, L. Bellaiche, and H. Fu, Nature 432, 737 (2004).

${ }^{38}$ I. Naumov and A. M. Bratkovsky, Phys. Rev. Lett. 101, 107601 (2008).

${ }^{39}$ K. C. Pitike, J. Mangeri, H. Whitelock, T. Patel, P. Dyer, S. P. Alpay, and S. Nakhmanson, J. Appl. Phys. (2018), in press; DOI: $10.1063 / 1.5037163$.

${ }^{40}$ Z. Cai, X. Wang, B. Luo, W. Hong, L. Wu, and L. Li, Comp. Sci. and Tech. 145, 105 (2017).

${ }^{41}$ Y. L. Li, S. Y. Hu, Z. K. Liu, and L.-Q. Chen, Appl. Phys. Lett. 78, 3878 (2001).

${ }^{42}$ J. Hlinka, Ferroelectrics 349, 49 (2007).

${ }^{43}$ Y. Li, S. Hu, Z. Liu, and L. Chen, Acta Mater. 50, 395 (2002).

${ }^{44}$ J. Hlinka and P. Marton, Phys. Rev. B. 74, 104104 (2006).

${ }^{45}$ D. Gaston, C. Newman, G. Hansen, and D. Lebrun-Grandié, Nucl. Eng. Design 239, 1768 (2009).

${ }^{46}$ Ferret is available at bitbucket.org/mesoscience/ferret.

${ }^{47}$ R. G. P. McQuaid, A. Gruverman, J. F. Scott, and J. M. Gregg, Nano Lett. 14, 4320 (2014).

${ }^{48}$ B. Lee, S. M. Nakhmanson, and O. Heinonen, Appl. Phys. Lett. 104, 262906 (2014).

${ }^{49}$ Y. Zhou, E. Iacocca, A. A. Awad, K. Dumas, F. C. Zhang, H. B. Braun, and J. Åkerman, Nature Comm. 6, 8193 (2015).

${ }^{50}$ R. P. Boardman, H. Fangohr, S. J. Cox, A. V. Goncharov, A. A. Zhukov, and P. A. J. de Groot, J. Appl. Phys. 95, 11 (2005). 